\section{Bisphosphonate verbessern Brustkrebsprognose}

\author{
Bei Brustkrebs im Frühstadium können Bisphosphonate das Risiko senken, \\ Rezidive zu erleiden und an den Folgen des Tumors zu sterben. Der Nutzen \\ beschränkt sich aber auf Frauen nach der Menopause.
}

\begin{abstract}
Dising ie Early Breast Cancer Trialists' Collaborative Group (EBCTCG) untersuchte mögliche onkologische Effekte einer Bisphosphonat-Therapie in einer Metaanalyse von 26 Studien. Die 18.766 Teilnehmerinnen hatten randomisiert für 2-5 Jahre ein Bisphosphonat oder Placebo erhalten. Während der Nachbeobachtungszeit von 5,6 Jahren war es bei 3.453 Frauen zu einem ersten Rezidiv gekommen, das bei 2.106 zum Tode führte. Für alle Patientinnen zusammen war nur ein grenzwertig signifikanter Vorteil durch eine Bisphosphonat-Therapie festzustellen, was das 10-Jahres-Risiko für Fernmetastasen, brustkrebsspezifische Mortalität und Gesamtmortalität betraf. Dabei war der Rückgang von Fernmetastasen hauptsächlich auf eine geringere Rate an Knochenmetastasen zurückzuführen. Auf Lokalrezidive oder den Be-
\end{abstract}

fall der kontralateralen Brust hatten Bisphosphonate keinen Einfluss.

Entscheidend für den Nutzen waren Alter bzw. Hormonstatus: Bei Frauen vor der Menopause waren die Medikamente in allen untersuchten Endpunkten nutzlos, bei postmenopausalen Frauen gingen sie dagegen mit hochsignifikanten Verbesserungen einher. Das 10-JahresRisiko für ein Rezidiv wurde bei Letzteren um $14 \%$ reduziert, das für Fernmetastasen und einen brustkrebsbedingten Tod jeweils um $18 \%$. Absolut wurden das Risiko für Knochenmetastasen um 2,2\% (6,6 vs. $8,8 \%)$ und die Brustkrebsmortalität um 3,3\% (14,7 vs. $18,0 \%)$ reduziert.

Fazit: Bei postmenopausalen Brustkrebspatientinnen wird durch eine Bisphosphonat-Therapie das krebsspezifische Überleben hochsignifikant verbes-

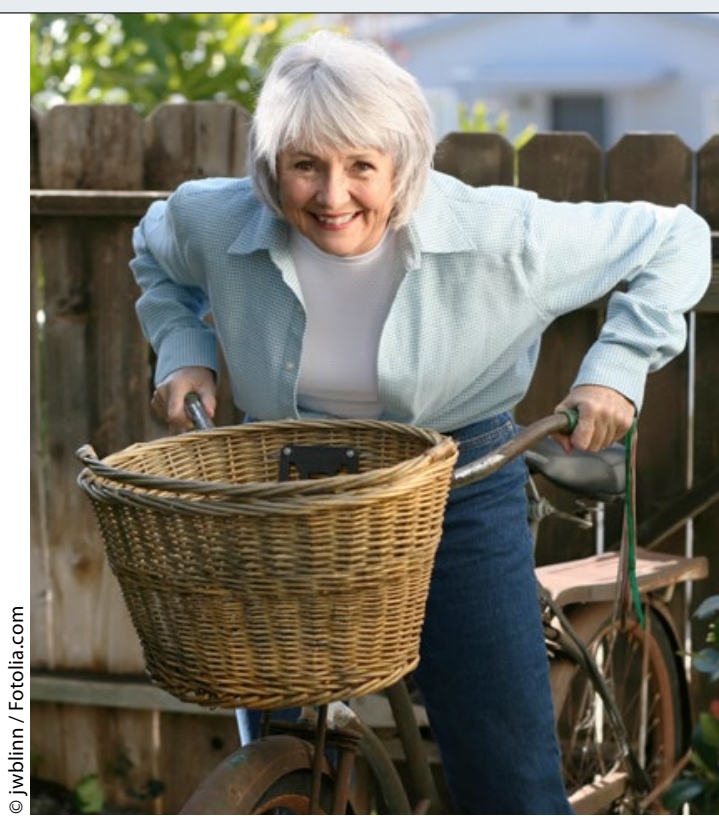

Ältere und postmenopausale Frauen profitierten deutlich von Bisphosphonaten.

sert. Die EBCTCG rät daher, den Einsatz von Bisphosphonaten „bei einem breiteren Spektrum dieser Patientinnen in Erwägung zu ziehen“. Beate Schumacher

EBCTCG. Adjuvant bisphosphonate treatment in early breast cancer: meta-analyses of individual patient data from randomized trials. Lancet. 2015;386(10001):1353-61.

\section{Palliative Chemotherapie bei Brustkrebs: Neue Substanzen nicht besser als Paclitaxel}

\begin{abstract}
Welches ist das optimale Vorgehen bei Patientinnen mit chemotherapienaivem, fortgeschrittenem Mammakarzinom? Dieser Frage ging die Cancer and Leukemia Group B (CALGB) in einer randomisierten Phase-III-Studie nach.
\end{abstract}

Ratio [HR] 1,59; p < 0,001). Auch für nab-Paclitaxel zeigte sich ein Trend zur Unterlegenheit verglichen mit Paclitaxel (medianes PFS: 9,3 Monate; HR 1,20; $\mathrm{p}=0,54)$. Subgruppenanalysen für $\mathrm{Pa}-$ tientinnen mit triple-negativer oder hormonrezeptorpositiver Erkrankung bestätigten die Unterlegenheit von Ixabepilon und nab-Paclitaxel. Die Ergebnisse zum Gesamtüberleben waren vergleichbar.

Hämatologische und nicht hämatologische Toxizitäten (z.B. Neutropenie, Anämie, periphere Neuropathie, Fatigue, Hypertonie, Schmerzen, Übelkeit) nahmen unter nab-Paclitaxel im Vergleich zu Paclitaxel zu, sodass Dosisreduktio- nen häufiger und früher erforderlich waren. Hämatologische Toxizitäten vom Grad $\geq 3$ traten unter Paclitaxel bei $22 \%$ der Patientinnen auf, unter nab-Paclitaxel bei $55 \%$ und unter Ixabepilon bei $12 \%$. Nicht-hämatologische Toxizitäten waren bei $49 \%$, $65 \%$ bzw. $58 \%$ der Patientinnen zu beobachten.

Fazit: Bei Patientinnen mit chemotherapienaivem, fortgeschrittenem Mammakarzinom waren neuere Substanzen wie Ixabepilon und nab-Paclitaxel nicht besser wirksam als Paclitaxel, wiesen aber eine höhere Toxizität auf, besonders nabPaclitaxel. Daher bleibt Paclitaxel 1-mal pro Woche die bevorzugte palliative Chemotherapie für diese Patientinnen.

Judith Neumaier

Rugo HS et al. Randomized Phase III Trial of Paclitaxel Once Per Week Compared With Nanoparticle Albumin-Bound Nab-Paclitaxel Once Per Week or Ixabepilone With Bevacizumab As First-Line Chemotherapy for Locally Recurrent or Metastatic Breast Cancer: CALGB 40502/NCCTG N063H (Alliance). J Clin Oncol. 2015;33(21):2361-9. 